\title{
Word-Initial Irregular Phonation as a Function of Speech Rate and Vowel Quality in Hungarian
}

\author{
Alexandra Markó ${ }^{1,2(\bowtie)}$, Andrea Deme ${ }^{1,2}$, Márton Bartók ${ }^{1,2}$, \\ Tekla Etelka Gráczi ${ }^{1,3}$, and Tamás Gábor Csapó ${ }^{1,4}$ \\ ${ }^{1}$ MTA-ELTE "Lendület” Lingual Articulation Research Group, \\ Budapest, Hungary \\ 2 Department of Phonetics, Eötvös Loránd University, Budapest, Hungary \\ ${ }^{3}$ Research Institute for Linguistics, Hungarian Academy of Sciences, \\ Budapest, Hungary \\ ${ }^{4}$ Department of Telecommunication and Media Informatics, \\ Budapest University of Technology and Economics, Budapest, Hungary \\ \{marko.alexandra, deme. andrea\}@btk.elte.hu, \\ bartokmarton@gmail.com, \\ graczi.tekla.etelka@nytud.mta.hu, csapot@tmit.bme.hu
}

\begin{abstract}
We examined vowel-initial irregular phonation in real words as a function of vowel quality, backness and height, and speech rate in Hungarian. We analyzed two types of irregular phonation: glottalization and glottal stop. We found that open vowels elicited more irregular phonation than mid and close ones, but we found no effect of the backness. The frequency of irregular phonation was lower in fast than in slow speech. Inconsistently with the claims of earlier studies, the relative frequency of glottalization to glottal stops was not influenced by speech rate in general. However, while /i/ was produced with a relatively higher ratio of glottal stops in fast speech, the open vowels showed the widely documented tendency of being realized with relatively less glottal stops under the same conditions.
\end{abstract}

Keywords: Irregular phonation · Glottal stop $\cdot$ Glottalization $\cdot$ Vowel height Vowel backness $\cdot$ Speech rate

\section{Introduction}

Irregular phonation is an umbrella term in the literature, which covers several realization types of irregularity in vocal fold vibration. Other terms like laryngealization, glottalization, creaky voice, etc. are also used, and in several cases they refer to only more or less similar domains of irregularity. Based on their formal characteristics, some authors use more accurate definitions for the subtypes, (e.g., Batliner et al. 1993, Dilley et al. 1996), while in several studies the concept of irregularity is introduced in a more intuitive manner. Considering the terminological variability in the literature, it is crucial that we clarify our use of terms in the present work. We refer to irregularity in general using the term irregular phonation, and we adopt the definition formed by Surana and 
Slifka (2006: p. 693): "A region of phonation is an example of irregular phonation if the speech waveform displays either an unusual difference in time or amplitude over adjacent pitch periods that exceeds the small-scale jitter and shimmer differences, or an unusually wide-spacing of the glottal pulses compared to their spacing in the local environment, indicating an anomaly with respect to the usual, quasiperiodic behavior of the vocal folds."

In the present study, we investigate two easily distinguishable types of irregular phonation. For one of these phenomena we apply the term glottalization (covering several possible subtypes) to refer to cases where irregularity can be observed as consecutive periods in voicing differing evidently in terms of duration, amplitude, or both. The second phenomenon is the unique glottal gesture which we refer to as glottal stop (Fig. 1.). As Esling and Harris (2005) pointed out, a single isolated burst (or set of aperiodic bursts) "may differ in timing from creaky voice but not in laryngeal configuration" (372). According to their interpretation, glottalization and glottal stop are articulated similarly; and several studies do also consider these phenomena various forms of the same glottal behavior (see e.g., Kohler 2001, Malisz et al. 2013).
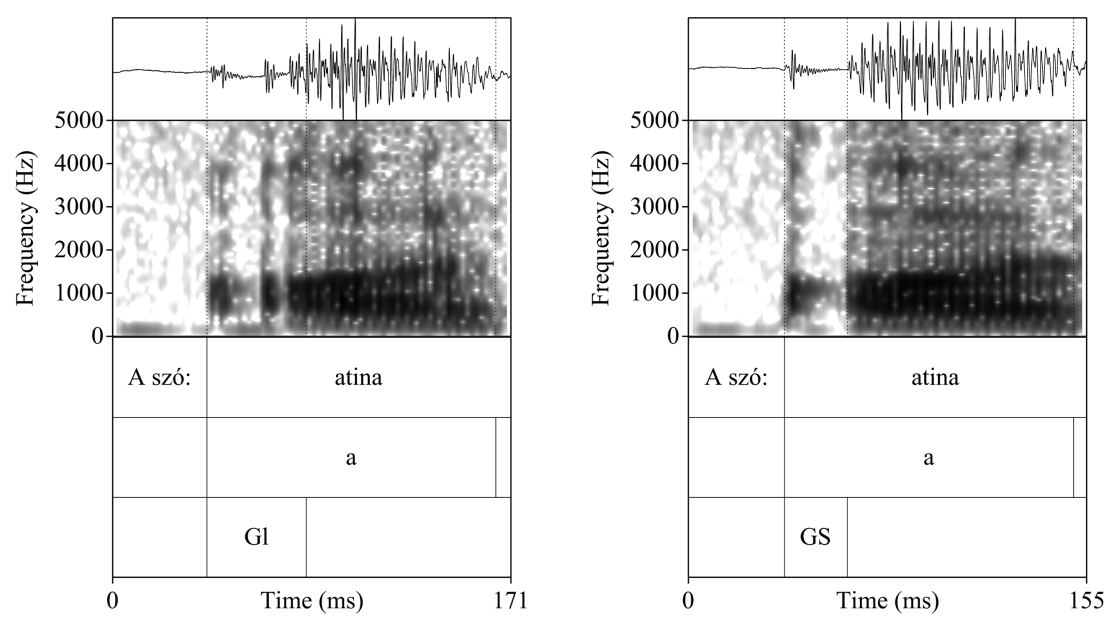

Fig. 1. Examples of glottalization (left) and glottal stop (right)

Irregular phonation serves prosodic functions in typologically unrelated languages, e.g., American English (Dilley et al. 1996), Czech, Spanish (Bissiri et al. 2011), German, Polish (Kohler 2001; Malisz et al. 2013), Hungarian (Markó 2013) and others. The occurrence of irregularity may be influenced by several factors (see some of these below), and it shows high inter- and intraspeaker variability (e.g., Dilley et al. 1996; Redi and Shattuck-Hufnagel 2001).

Kohler (2001: pp. 282-285) defined four types of irregular phonation (which he labelled as glottalization covering "the glottal stop and any deviation from canonical modal voice”) as follows. (1) Vowel-related glottalization phenomena which signal the boundaries of words or morphemes. (2) Plosive-related phenomena which occur as 
reinforcement or even replacement of plosives. (3) Syllable-related phenomena which characterize syllable types along a scale from a glottal stop to glottalization (e.g., Danish stød). (4) Utterance-related phenomena which comprise (i) phrase-final relaxation of phonation, and (ii) truncation glottalization, i.e., utterance-internal tensing of phonation at utterance breaks.

Vowel-initial irregular phonation (a specific case of type (1) above) was analyzed in several studies. Malisz et al. (2013) examined the conditioning effect of speech style (speech vs. dialogue), presence of prominence, phrasal position (initial vs. medial), speech rate, word type, preceding segment, and following vowel height on the occurrence frequency of word-initial glottalization in Polish and German. They concluded -among others- that vowels bearing prominence were more frequently marked glottally (in both languages), faster rates reduced glottal marking in general, especially the occurrence frequency of glottal stops, but faster rates increased the relative frequency of occurrence of glottalization. They also found that low vowels were more frequently glottalized in both languages than non-low vowels; however it must also be noted that speech rate and vowel quality factors were not systematically varied in this study.

Lancia and Grawunder (2014) used pseudo-words to facilitate vowel-initial irregular phonation, and to analyze the conditioning factors of vowel height (high vs. low: /i/ vs. /a/), the presence of stress, and the place of articulation of the preceding consonants. They concluded, that retracted tongue body (i.e., low-back tongue position) favors the production of irregular phonation (particularly strongly in unstressed syllables). It should be noted, however, that in the cited study the high-front /i/ and the low-back /a/ were compared, thus the results might have revealed an interaction effect of the features vowel height and backness. Lancia and Grawunder did not treat the vowel height and backness as separate factors, as they aimed to analyze the effect of the maximally retracted tongue position using /a/. Therefore, the cited results are not informative regarding the possible independent effect of the tongue height and the front-back dimension.

In Hungarian a systematic analysis of the effect of speech rate and vowel quality in vowel-initial irregular phonation has not been carried out so far; in addition, to the authors' knowledge, a study considering the interaction of these factors is also nonexistent for any other languages either. Moreover, in earlier studies regarding vowel-related irregularity in Hungarian (e.g., Markó 2013), glottalization and glottal stops were not treated separately. Therefore no data is available on the relative frequency of these two types either.

In the present study we investigated the effect of vowel height and backness, and speech rate, using a balanced speech material, which was also well controlled in terms of speech rate. In the analysis, two types of irregular phonation (glottalization and glottal stop) were also taken into account.

Based on previous results for other languages we addressed the following questions. Is irregular phonation more frequent in word-initial vowels in Hungarian if (i) the speech rate is slow (as opposed to fast); (ii) the vowel is back (as opposed to front); (iii) the vowel is open (as opposed to close or close-mid)? (iv) Do glottal stops occur less frequently in fast speech, while the relative amount of glottalization increases? We hypothesized that the occurrence frequency of irregular phonation in general is higher 
in slow speech than in fast speech. Furthermore, we assumed that back vowels elicit irregular phonation in a higher ratio than front ones both in slow and fast speech, and that open vowels favor irregular phonation more than non-open (close or close-mid) ones. Finally, we also assumed that faster speech rate reduces the amount of glottal stops, but increases the relative frequency of glottalization.

\section{Material and Method}

\subsection{Material and Experiment Design}

The test material consisted of disyllabic Hungarian pronominal adverbs: innen /in: $\varepsilon$ / 'from here'; onnan /on:pn/ 'from there'; ennek /عn:ck/ 'for this'; annak /pn:pk/ 'for that'. These adverbs start with four different vowel qualities which vary both in the vowel height (close /i/ vs. mid /o/ vs. open $/ \varepsilon \mathrm{p} /$ ) and the backness (back /o $\mathrm{p} / \mathrm{vs}$. front /i $\varepsilon /$; while backness also co-varies with lip spreading) (see Fig. 2). (It is important to note here that in the present study by the introduction of $/ \varepsilon /$ as an open vowel we used a "simplified" feature set along the vowel height dimension to which the system described by Szende (Fig. 2) and the similar "acoustic openness" of $/ \varepsilon /$ and /p/ provided the basis.)

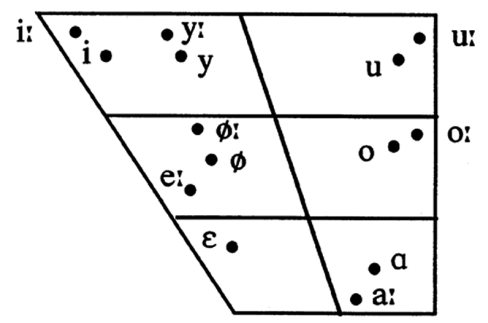

Fig. 2. The Hungarian vowel inventory (Szende 1994: p. 92) (Please note, that even though in this system Szende labels the / $/ \mathrm{q}$ quality without the lip-rounding gesture, i.e., as / $\mathrm{a} /$, the rounded $/ \mathrm{p} /$ is a more generally accepted transcription of the sound in question).

These target words were embedded in the following phrase: Mondd: [target word] kell. 'Say: [target word] needed'. All of the word-initial target vowels bear sentencial accent on the first syllable (given that word stress is fixed on the first syllable in Hungarian).

The stimuli were presented on a computer screen. Each trial consisted of two display screens: first the introductory part (Mondd:) was showed to the participant, then the target item (target word + kell) was displayed. The participants' task was to read aloud the target item, but not the introductory part.

In order to elicit speech rate differences between the conditions, the timing of the display screens was manipulated. In the "slow" speech condition, each display screen appeared for $1500 \mathrm{~ms}$ resulting in $3000 \mathrm{~ms}$ for one trial in total (including the introductory part and the "target word + kell" construction). In the "fast" speech condition 
the timer was set to $500 \mathrm{~ms}$, resulting in $1000 \mathrm{~ms}$ for one trial in total. (During the recordings several other timer settings were also applied, and the setting to serve as the "fast" condition was selected posterior to the recordings on the basis of the speakers' ability to produce the items properly, i.e., separately and without errors.) As the timing of the introductory part reflected also the timing of the target item (target word + kell), it enabled the speakers to prepare for the production of the latter one.

The trials were ordered into blocks: within each block all the four different target words occurred in a randomized order once, and these blocks were repeated 5 times consecutively for each ("slow" and "fast") condition. First the "slow" condition, then the "fast" condition was recorded in the case of every participant. 4 vowel qualities/ target words $\times 5$ repetitions $\times 2$ speech rate conditions, i.e., 40 vowels per speaker were recorded. The recordings were made in a sound-treated booth, using a tie-clip omnidirectional condenser microphone and an external soundcard.

\subsection{Participants}

Previous results revealed that Hungarian female speakers tend to produce irregular phonation more frequently than male speakers (see e.g., Markó 2013); therefore in the present study only female speakers were included; all 33 of them were university students, and native speakers of Hungarian, who reported no hearing or speech deficits. In order to ensure that the "slow" and the "fast" conditions differentiate properly (i.e., they may be differentiated by a conceptually sound value), a threshold for the speech rate difference was introduced (see below, in 2.4).

This threshold was not exceeded by the data in the case of 15 participants, thus finally in the main analysis 18 speakers' material was involved. The speakers' age ranged between 19 and 34 years, with a mean of 24.9 years.

In the "slow" condition 359 vowels were analyzed (as one speaker mispronounced one target word), while in the "fast" condition the number of analyzed vowels was 360 .

\subsection{Annotation}

The "target word + kell" construction, the word-initial vowel, and the irregular phonation at the beginning of the word-initial vowel were labelled manually in Praat (Boersma and Weenink 2016). The vowel qualities were labelled automatically (on the basis of the stimuli order), and then the quality label assignment was also confirmed manually as correct by the annotators (two of the authors of the present paper) auditorily. In the case of mispronunciation or any other errors involving the production of the vowel of interest, the vowel was excluded from the material. Vowel boundaries were defined on the basis of the $\mathrm{F}_{2}$ trajectory.

The labeling of the irregular phonation was performed in accordance with the methodology proposed by previous studies (e.g., Dilley et al. 1996; Bőhm and Ujváry 2008) in which visual (waveform and spectrogram) and auditive information was combined. A given vowel was labelled as irregular if (i) its first consecutive $\mathrm{f}_{0}$-periods differed evidently in terms of duration, amplitude, or both (these cases were marked as 
glottalization, see Fig. 1, left), or if (ii) one (or more) glottal stop was observed at the beginning of the vowel (these cases were marked as glottal stop, see Fig. 1, right) (see Dilley et al. 1996). Those cases where glottal stops were followed by creaky voice were labelled as glottalization. The occurrences of irregular phonation were noted by one listener, who marked the entire corpus in Praat. In the case of ambiguous occurrences the pitch curve and pulses shown by Praat (standard settings) were also taken into consideration.

We analyzed the ratio of vowels produced with irregular phonation with respect to vowel quality, vowel height and backness, and speech rate. We also determined and compared the ratio of glottalized vowel occurrences to the number of vowels realized with glottal stops in the two speech rate conditions.

\subsection{Control of Speech Rate}

Regarding that one of the aims of the present study was making a comparison between "slow" and "fast" speech, it was inevitable to carefully control the difference of the speech rate between these two conditions. To achieve this goal, first we manipulated the timing of the display screens (see above). And second, we also set a perceptually motivated threshold for the speech rate differences.

In the case of Hungarian, the just noticeable difference (JND) for speech tempo has not been studied so far; however, there are JND data for other languages which may be taken as a reasonable reference for Hungarian as well. For instance, for Dutch speech fragments Quené (2007) found 5\% JND for artificially increased/decreased speech rate differences, while he also noted that this value may be an overestimation, and that in the case of everyday communicative situations, the JND is probably lower.

As in our case the number of the phonemes per item was constant, to calculate speech rate differences, not the speech sound per duration values, but only the item durations were measured in the two speech rate conditions.

The target item durations were measured and compared both in "slow" and "fast" conditions speakerwise. However, as in our study five repetitions of each item were analyzed and averaged, we decided to apply a higher threshold of $10 \%$ to account for the expected reduction in duration variability. As mentioned above, there were 18 speakers who differentiated their speech rates by more than $10 \%$ on average; therefore these 18 speakers' data were included in the main analysis. The duration difference between the two conditions ranged between $9.6 \%$ and $28.5 \%$ speakerwise, and the mean of differences was $16.8 \pm 5.5 \%$. The overall item duration was $852 \pm 107 \mathrm{~ms}$ in the "slow" condition, and $705 \pm 69 \mathrm{~ms}$ in the "fast" condition (for all 18 speakers).

\subsection{Statistical Analyses}

Three 2-way repeated measures ANOVAs were performed with the factors vowel quality and speech rate, vowel backness and speech rate, and vowel height and speech rate, and a confidence level set to $95 \%$. 


\section{Results}

\subsection{Vowel-Initial Irregular Phonation as a Function of Vowel Quality and Speech Rate}

The ratio of vowels produced with irregular phonation (pooled over speakers) as a function of vowel quality and speech rate is presented in Fig. 3.

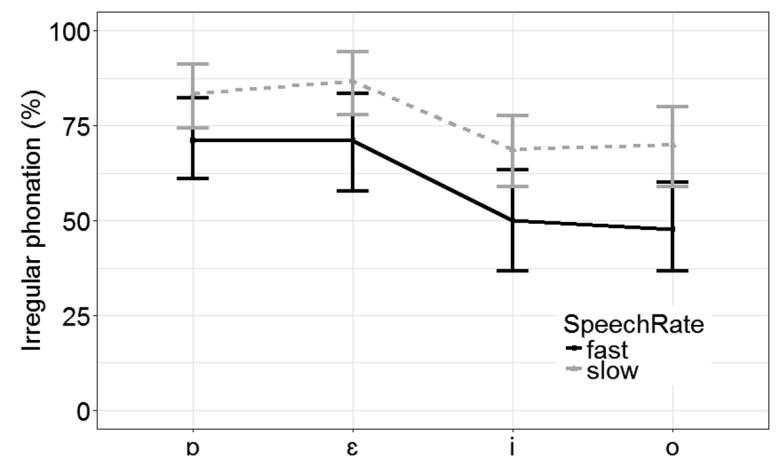

Fig. 3. The ratio of vowels produced with any kind of irregular phonation as a function of vowel quality and speech rate $($ mean $+95 \% \mathrm{CI})$

In the "slow" condition the ratio of vowel-initial irregular phonation in the front and close /i/ was $68.6 \pm 20.7 \%$, while in the "fast" condition it was $50.0 \pm 30.1 \%$ of all cases. In the case of the front and open vowel / $/$ / these ratios were $86.7 \pm 18.1 \%$ in the "slow" and $71.1 \pm 29.3 \%$ in the "fast" conditions. The back and mid vowel /o/ was produced with irregular phonation $70.0 \pm 24.0 \%$ of all cases in the "slow" and $47.8 \pm 25.8 \%$ of all cases in the "fast" condition. Finally, the back and open $/ \mathrm{p} /$ showed $83.3 \pm 18.5 \%$ irregular occurrences in the "slow" and $71.1 \pm 24.9 \%$ in the "fast" condition.

The ANOVA showed significant main effects of both speech rate $(F(1$, $17)=17.38, p<0.001)$ and vowel quality $(F(3,51)=10.56, p<0.001)$, but the interaction of these factors turned out to be non-significant.

\subsection{Vowel-Initial Irregular Phonation as a Function of Vowel Backness and Speech Rate}

The ratio of vowels produced with irregular phonation as a function of vowel backness and speech rate is shown in Fig. 4. The back /o/ and /p/ and the front $/ \varepsilon /$ and $/ \mathrm{i} /$ vowels were produced with irregular phonation in a similar ratio both in the "slow" and the "fast" conditions. In the "slow" condition front vowels showed $77.6 \pm 21.2 \%$ ratio of irregular occurrences, while back vowels showed $76.7 \pm 22.1 \%$ of all cases. In the "fast" condition the ratios were $60.6 \pm 31.2 \%$ and $59.4 \pm 27.7 \%$, respectively. Regarding this comparison, statistical analysis showed a significant difference between 
the speech rate conditions $(F(1,68)=14.58, p<0.001)$ but not between the front and back vowel groups.

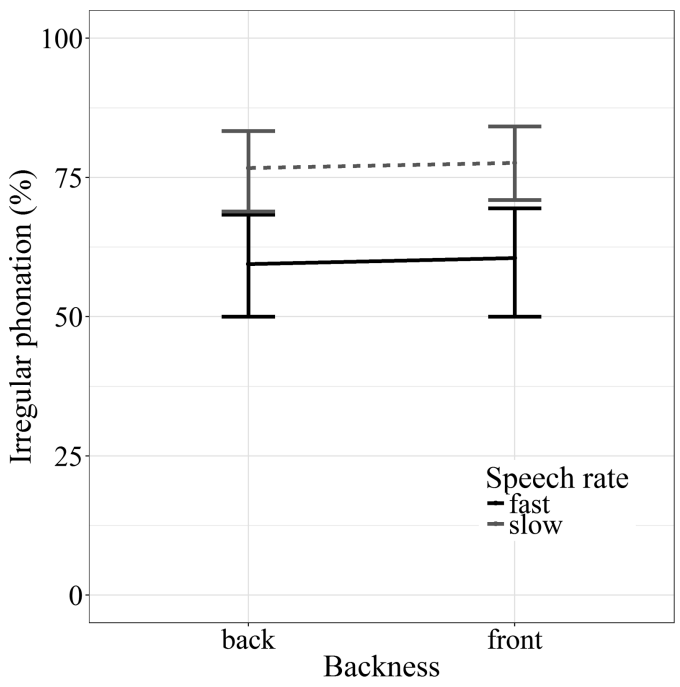

Fig. 4. The ratio of vowels produced with any kind of irregular phonation as a function of vowel backness and speech rate $($ mean $+95 \% \mathrm{CI})$

\subsection{Vowel-Initial Irregular Phonation as a Function of Vowel Height and Speech Rate}

The ratio of vowel realizations with irregular phonation in terms of vowel height and speech rate are shown in Fig. 5. The close vowel /i/ was produced with irregular phonation $68.6 \pm 20.7 \%$ of all tokens in the "slow" and $50.0 \pm 30.1 \%$ of all tokens in the "fast" condition. The mid vowel /o/ showed vowel-initial irregular phonation $70.0 \pm 24.0 \%$ of all cases in the "slow" and $47.8 \pm 25.8 \%$ in the "fast" condition. The open vowels $/ \mathrm{p} /$ and $/ \varepsilon /$ were produced with irregular phonation at the highest ratio: $85.0 \pm 18.1 \%$ in the "slow" and $71.1 \pm 26.8 \%$ in the "fast" condition.

According to the ANOVA, there was no significant interaction between speech rate and vowel height, but both factors had a significant main effect: vowel height: $F(2$, $34)=13.20, p<0.001$; speech rate: $F(2,17)=17.28, p<0.001$.

\subsection{Ratio of Glottalization and Glottal Stops as a Function of Vowel Quality and Speech Rate}

The ratio of occurrences of glottal stops and glottalization as a function of vowel quality and speech rate are presented in Fig. 6. Relative to the number of all vowel realizations (not displayed on Fig. 6.) both in the "slow" and the "fast" conditions the percentage of glottalization (42.6\% and $35.0 \%$ of all occurrences, respectively) exceeded the percentage of glottal stops $(34.5 \%$ and $25.0 \%$ of all occurrences, 


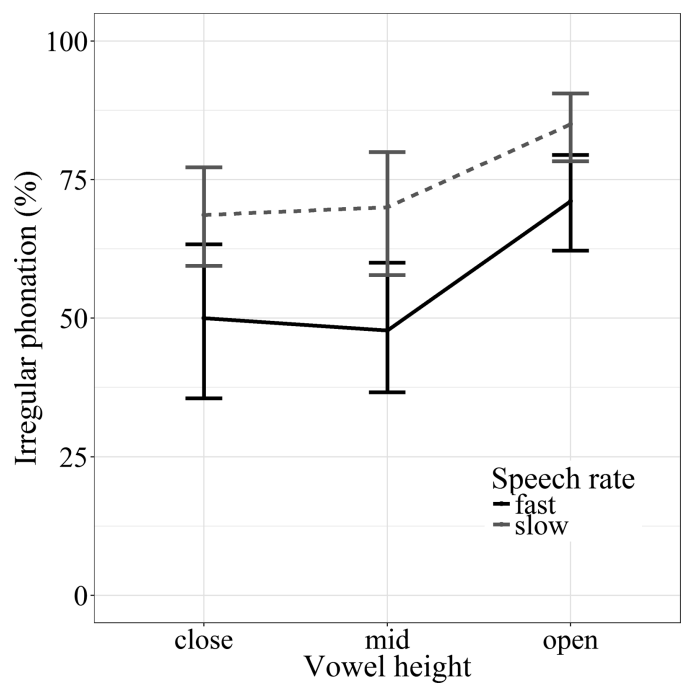

Fig. 5. The ratio of vowels produced with any kind of irregular phonation as a function of vowel height and speech rate $($ mean $+95 \% \mathrm{CI})$

respectively). Relative to the number of all irregular occurrences both in the "slow" and the "fast" conditions the percentage of glottalization $(54.9 \%$ and $56.0 \%$ of all irregular occurrences, respectively) exceeded the percentage of glottal stops $(45.1 \%$ and $43.4 \%$ of all irregular occurrences, respectively) as well (see Fig. 6).

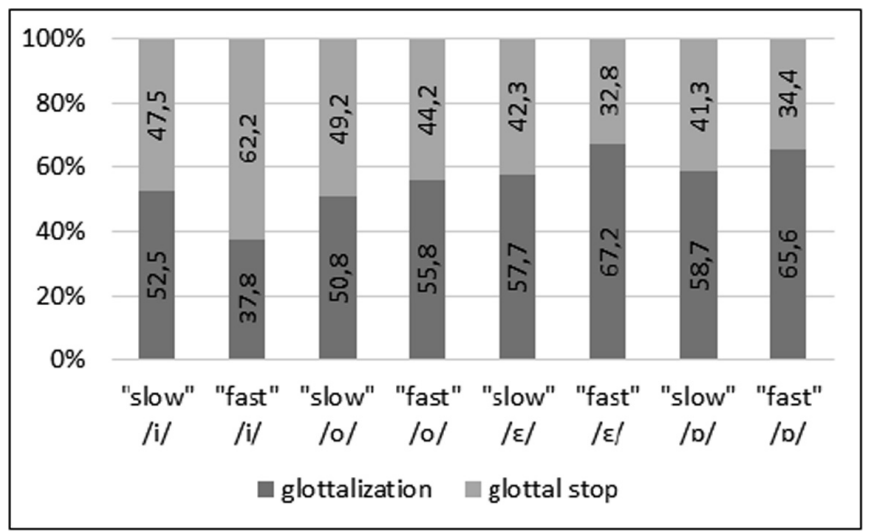

Fig. 6. The ratio of the two types of irregular phonation (relative to all irregular occurrences) as a function of vowel quality and speech rate

The ratio of glottalization and glottal stops were close to equal in the case of $/ \mathrm{i} / \mathrm{in}$ the "slow" condition, while in the "fast" condition the ratio of glottal stops was well above the ratio of glottalization. For the "slow" condition the pattern was very similar 
in the case of /o/; in the "fast" condition, however, glottalization was relatively more frequent than glottal stops. In the case of $/ \varepsilon /$ and $/ \mathrm{p} /$, the ratio of glottalization exceeded the ratio of glottal stops in both of the conditions. Although we observed differences in the glottalization to glottal stop ratio between the two conditions in three of the four analyzed vowels, the close /i/ showed the opposite tendency with the change in speech rate, than that observed in the case of open vowels. The direction of the change in the mid /o/ was the same as in the case of the open vowels, but the degree of change was smaller.

\section{Discussion and Conclusion}

Our first hypothesis, claiming that the occurrence frequency of irregular phonation is higher in slow than in fast speech, has been confirmed. Our second and third hypotheses were partially confirmed by the data: even though vowel backness did not have an effect on vowel-initial irregular phonation, we showed that open vowels favor irregular phonation more than mid and close ones both in slow and fast speech. Our fourth assumption claiming that faster speech rates reduce the relative amount of glottal stops, while increasing the frequency occurrence of glottalization was not verified, since glottalization was more frequent in both speech rates in general. However, to some extent, at the different vowel heights studied, different tendencies were found.

Considering that in the present study the effect of phonetic position, vowel quality, and speech rate were strictly controlled and investigated in real words, we can conclude that open vowels tend to elicit more irregular phonation than mid and close ones, irrespective of the vowel backness. We can also conclude that the frequency of irregular phonation tends to be lower in fast than in slow speech (or at least in speech accelerated under laboratory conditions). Relative frequency of glottalization to glottal stops in phrase-initial vowel-initial position did not appear to be influenced by speech rate in general, which itself was inconsistent with the claims of earlier studies (e.g., Malisz et al. 2013). However, taking the analyzed vowels separately into account, we observed that the behavior of the close /i/ was opposite to that of the open /p/ and $/ \varepsilon /$. While the open vowels showed the widely documented tendency of being realized with relatively less glottal stops in fast speech, /i/ was produced with a relatively higher ratio of glottal stops under the same conditions. This result suggests that the vowel height has an effect not only on the frequency of irregular phonation, but also on the manner of its realization in the case of word- and phrase-initial vowels.

As already mentioned in the Introduction section, Lancia and Grawunder (2014) reported that the effect of laryngealization was found to be weaker when the tongue was more fronted (in the case of /i/) and that laryngealization was produced with a retraction of the tongue (in the case of /a/). They explained this finding as a result of an interaction between the tongue position and the laryngeal settings, namely that higher tongue positions also increase the vertical position of the larynx, which then modifies the mode of phonation (lower positions leading to more lax, while higher positions leading to more tense phonation). However, as we also pointed out, the vowel height and backness features were not considered as separate factors in the cited study, thus their possible individual effects were not revealed. In the present study, we aimed to 
tease apart these factors, and we showed that it is the vowel height feature that exhibits an effect on the occurrence of irregular phonation, as the low back /p/ and the low front $/ \varepsilon /$ vowels showed similarly high ratio of irregular occurrences, irrespective of their backness feature. Interpreted in the light of the argumentation of Lancia and Grawunder (2014), we thus conclude that it may be the vowel height that is the most influential vocalic feature on the vertical position of the larynx, and thus indirectly it is also the most influential with respect to the mode of phonation (at least as far as the occurrence of word-initial irregular phonation is considered).

This claim is also supported by previous articulatory studies that suggest, that front close unrounded vowels (e.g., /i/, /e/) have a higher larynx position than back close, close-mid or open rounded vowels (as /u/, /o/, /p/) (Hess 1998, Hoole and Kroos 1998, Demolin et al. 2002). However, as Esling (2005: p. 23) points out, from an articulatory perspective, the difference between "openness" categories should also be considered fundamentally different in "front" than in "back" vowels, as in "front" vowels it is indeed the difference in jaw opening, while in "back" vowels it is raising in the case of "close", and retraction of the tongue in the case of "open" vowels. And on this basis, Esling (2005) also suggests that due to the connections of the tongue muscles to other speech organs, the "back open" /p/-like vowels, should or might exhibit the strongest connection to (and the greatest effect on) the laryngeal settings via the direct link of the hyoid bone. Even though we also found that high vowels are less likely to facilitate the occurrence of irregular phonation, the latter prediction, i.e. the special effect of low/back vowels, was not confirmed by our data. To clarify the question, if the backness feature of the vowels does indeed not have a relevant effect on the occurrence frequency of irregular phonation, we plan to extend our study by the analysis of articulatory data of the vowels at hand.

Acknowledgement. The authors are grateful to Gergely Varjasi for his valuable help in recruitment of the participants and in conducting the experiments.

\section{References}

Batliner, A., Burger, S., Johne, B., Kiessling, A.: MÜSLI: a classification scheme for laryngealizations. In: Working Papers, Prosody Workshop, pp. 176-179. Schweden, Lund (1993)

Bissiri, M.P., Lecumberri, M.L., Cooke, M., Volín, J.: The role of word-initial glottal stops in recognizing English words. In: Proceedings of Interspeech 2011, pp. 165-168, Florence (2011)

Boersma, P., Weenink, D.: Praat: doing phonetics by computer. Version 6.0.17 (2016). http:// www.praat.org/

Bőhm, T., Ujváry, I.: Az irreguláris fonáció mint egyéni hangjellemző a magyar beszédben. Beszédkutatás 2008, 108-120 (2008)

Demolin, D., Hassid, S., Metens, T., Soquet, A.: Real-time MRI and articulatory coordination in speech. Comptes Rendus - Biologies 325(4), 547-556 (2002)

Dilley, L., Shattuck-Hufnagel, S., Ostendorf, M.: Glottalization of word-initial vowels as a function of prosodic structure. J. Phon. 24, 423-444 (1996) 
Esling, J.H.: There are no back vowels: The laryngeal articulator model. Can. J. Linguist. 50, 1344 (2005)

Esling, J.H., Harris, J.: States of the glottis: An articulatory phonetic model based on laryngoscopic observations. In: Hardcastle, W.J., Beck, J. (eds.) A Figure of Speech: A Festschrift for John Laver, pp. 347-383. Lawrence Erlbaum Associates, Mahwah (2005)

Hess, S.A.: Pharyngeal Articulations. Ph.D. dissertation, UCLA (1998)

Hoole, P., Kroos, C.: Control of larynx height in vowel production. In: Proceedings of the 5th Conference on Language Processing (ICSLP), vol. 2, pp. 531-534 (1998)

Kohler, K.J.: Plosive-related glottalization phenomena in read and spontaneous speech. A stød in German? In: Grønnum, N., Rischel, J. (eds.) To Honour Eli Fischer-Jørgensen, pp. 174-211. Kopenhagen, Reitzel (2001)

Lancia, L., Grawunder, S.: Tongue-larynx interactions in the production of word initial laryngealization over different prosodic contexts: a repeated speech experiment. In: Fuchs, S., Grice, M., Hermes, A., Lancia, L., Mücke, D. (eds.) Proceedings of the 10th (ISSP), pp. 245248, Cologne (2014)

Malisz, Z., Żygis, M., Pompino-Marschall, B.: Rhythmic structure effects on glottalisation: a study of different speech styles in Polish and German. Lab. Phon. 4(1), 119-158 (2013)

Markó, A.: Az irreguláris zönge funkciói a magyar beszédben. ELTE Eötvös Kiadó, Budapest (2013)

Quené, H.: On the just noticeable difference for tempo in speech. J. Phon. 35, 353-362 (2007)

Redi, L., Shattuck-Hufnagel, S.: Variation in the realization of glottalization in normal speakers. J. Phon. 29, 407-429 (2001)

Surana, K., Slifka, J.: Acoustic cues for the classification of regular and irregular phonation. In: INTERSPEECH-2006, paper 1755-Mon3FoP.1 (2006)

Szende, T.: Illustrations of the IPA: Hungarian. JIPA 24(2), 91-94 (1994) 